\title{
DIRECT MEASUREMENTS OF BOUND MAGNETIC POLARON MAGNETIZATION IN Ga-DOPED $\mathrm{Cd}_{1-x} \mathrm{Mn}_{x} \mathrm{Te}$
}

\author{
J. Stankiewicz* , F. Palacio
}

Instituto de Ciencia de Materiales de Aragón, Consejo Superior de Investigaciones Científicas and Universidad de Zaragoza, 50009-Zaragoza, Spain

\section{AND F. VILLUENDAS}

Departamento de Física Aplicada, Universidad de Zaragoza, 50009-Zaragoza, Spain

Light induced remanent changes in magnetization of $n$-type $\mathrm{Cd}_{1-x} \mathrm{Mn}_{x} \mathrm{Te}$ $(x=0.01,0.05)$ single crystals have been measured at $T=2$ and $5 \mathrm{~K}$ and in magnetic fields of up $0.5 \mathrm{~T}$. The effect observed gradually saturates with increasing magnetic field. It also correlates with light induced increase in shallow donor concentration measured on the same samples. The bound magnetic polaron theory accounts for the temperature and magnetic field variations of the persistent magnetization. There are no fitting parameters.

PACS numbers: 75.50.Pp

The formation of bound magnetic polarons (BMPs) in magnetic semiconductors is a consequence of a strong exchange interactions between localized spins of magnetic ions and spins of charge carriers. In it, charge carriers bound to impurities polarize the spins of magnetic ions within their orbit, thus lowering the total energy of the system. Effects related to BMPs have been observed in Raman scattering [1], photoluminescence [2] and electron transport [3] experiments in various diluted magnetic semiconductors (DMS). Different successful theoretical approaches have been developed to treat the BMP problem [4-6]. However, not all thermodynamic properties of BMP have been experimentally studied. Thus, direct magnetization measurements of persistent BMP have been reported for a limited range of magnetic field only once [7]. The contribution of BMP to the magnetization of a paramagnetic DMS is small and strong paramagnetic background in these materials makes such measurements difficult. Application of the persistent photoconductivity effect, observed in In- and Ga-doped CdMnTe crystals [8], helps to overcome this difficulty. Illumination of the sample at low temperatures raises (by several orders of magnitude) the shallow donor concentration which persists

\footnotetext{
*e:mail address: jolanta@posta.unizar.es
} 
for a long time after the light is shut off. BMPs, formed on light-induced donors, give rise to an additional magnetization which may be measured as the host magnetization does not change upon illumination otherwise. Such measurements have been reported for Ga-doped $\mathrm{Cd}_{1-x} \mathrm{Mn}_{x} \mathrm{Te}_{1-y} \mathrm{Se}_{y}$ crystals $(x=0.10)$ at 2.1 and $4.2 \mathrm{~K}$ in magnetic fields of up to $0.02 \mathrm{~T}$ and have been described by the BMP theory [7]. However, the range of magnetic field used in Ref. [7] has been too small to observe saturation of BMP magnetization with increasing magnetic field. Here, we report BMP magnetization studies in $\mathrm{Cd}_{1-x} \mathrm{Mn}_{x} \mathrm{Te}(x=0.01,0.05)$ single crystals doped with $\mathrm{Ga}$ in magnetic fields of up to $0.5 \mathrm{~T}$ at $T=2$ and $5 \mathrm{~K}$. Several new features of BMP behavior are observed which confirm the existing theoretical picture of these complexes.

Single crystals of $\mathrm{Cd}_{1-x} \mathrm{Mn}_{x} \mathrm{Te}$, doped with gallium, were grown by a modified Bridgman method. The as-grown alloys were of high resistivity, because of a large density of $\mathrm{Cd}$ vacancies. To increase $n$-type conductivity, $\mathrm{Cd}_{1-x} \mathrm{Mn}_{x} \mathrm{Te}: \mathrm{Ga}$ was annealed in $\mathrm{Cd}$ vapor at a temperature of $1000^{\circ} \mathrm{C}$ for $100 \mathrm{~h}$. The manganese concentrations were checked by photoluminescence measurements; they agreed well with the nominal $x$ values. Samples used in our study were cleaved from the bulk material. Hall effect measurements were carried out in a temperature range from 1.6 to $300 \mathrm{~K}$. We used unpolarized light emitting diodes $(\lambda=860 \mathrm{~nm}$ or $780 \mathrm{~nm})$ with an intensity of about $10 \mathrm{~W} / \mathrm{m}^{2}$ as a source of illumination at low temperatures. Excitation energy was below energy gap value. Magnetization measurements were performed using a commercial Quantum Design SQUID magnetometer with an optical fiber insert which was designed by us. After each experimental run, we heated the sample to about $150 \mathrm{~K}$ and then slowly cooled it down in order to reestablish the initial conditions.

The electron concentration variations with temperature in $\mathrm{Cd}_{1-x} \mathrm{Mn}_{x} \mathrm{Te}: \mathrm{Ga}$ $(x=0.01$ and 0.05$)$ samples before and after illumination are shown in Fig. 1 . The observed behavior can be explained in terms of metastability of Ga dopant [8].

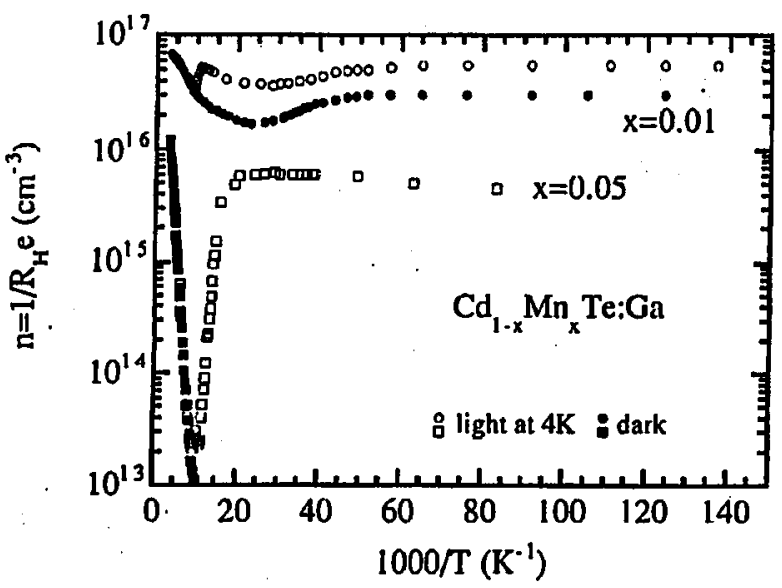

Fig. 1. Temperature dependence of the Hall concentration in $\mathrm{Cd}_{0.99} \mathrm{Mn}_{0.01} \mathrm{Te}$ :Ga and $\mathrm{Cd}_{0.95} \mathrm{Mn}_{0.05} \mathrm{Te}: \mathrm{Ga}$ in dark and after long illumination at $4 \mathrm{~K}$. 
Thus, for $T>100 \mathrm{~K}$, the dark carrier concentration decreases upon cooling down as electrons freeze-out on the relaxed deep donor levels. The slope of the reciprocal of the Hall coefficient versus $1 / T$ in this region gives an activation energy of the order of 7 and $90 \mathrm{meV}$ for $x=0.01$ and $x=0.05$, respectively. Below $\sim 100 \mathrm{~K}$, further electron freeze-out may occur only at the unrelaxed state of the defect with a smaller activation energy since the energy required to produce lattice distortion around the defect is greater than $k T$. Electrons excited by light from deep traps remain in the conduction band persistently, if their energy is lower than the capture barrier. The maximum photoinduced change in concentration of shallow donors, $\Delta N_{\mathrm{D}}$, estimated from the Hall effect measurements, is about $2.1 \times 10^{16} \mathrm{~cm}^{-3}$ and $4.1 \times 10^{15} \mathrm{~cm}^{-3}$ for $x=0.01$ and 0.05 , respectively.

Changes in the magnetization upon illumination in doped $\mathrm{Cd}_{1-x} \mathrm{Mn}_{x} \mathrm{Te}$ samples are shown in Fig. 2 as a function of applied magnetic field for two different temperatures: $T=2$ and $5 \mathrm{~K}$. Several samples of each composition were studied. The total change in magnetization (upon illumination that is long enough to reach saturation) is proportional to the mass of the samples and to light induced persistent donor concentration. Therefore, it is a measure of the magnetization of BMPs formed on shallow donors. The BMP magnetization gradually saturates as magnetic field increases. The saturation is faster at lower temperatures and for samples with larger Mn content. The BMP contribution to the magnetization, shown by solid curves in Fig. 2, has been calculated from the BMP partition function [5]. It can be equally obtained by taking the partial derivative of the donor-BMP free

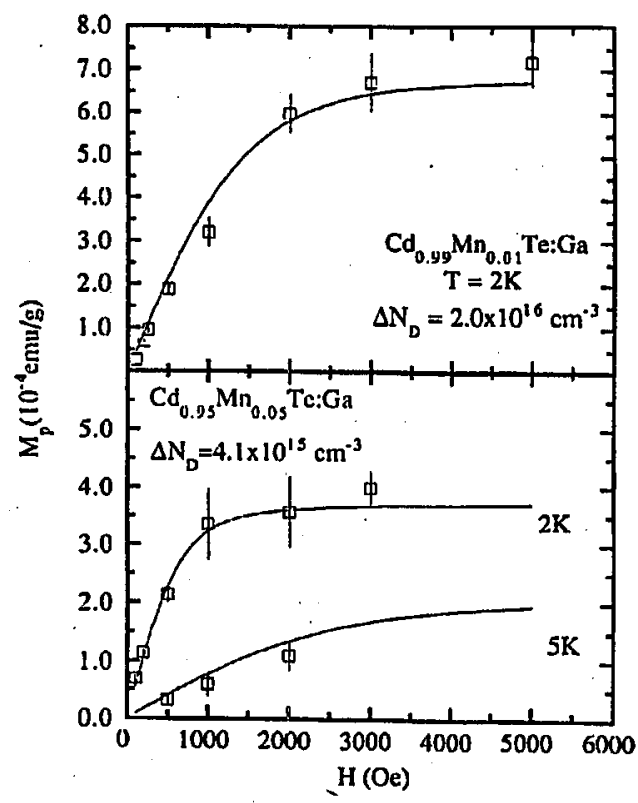

Fig. 2. Experimental change in the magnetization (open squares) and calculated BMP magnetization (solid lines) as a function of magnetic field for two different compositions at two temperatures, 2 and $5 \mathrm{~K}$. 
energy with respect to magnetic field [4]. We have included in our calculations the effect of the antiferromagnetic exchange coupling between the magnetic ions in the way proposed in Ref. [6]. A modified Brillouin function has been used to describe the magnetic properties of DMS. Phenomenologically, the parameter $T_{0}$ has been introduced in the description of DMS magnetization in Ref. [9]. The lattice temperature $T$ was replaced by the effective value $T_{\text {eft }}=T+T_{0}$, where $T_{0}$ is related to the exchange integrals involving non-nearest-neighbor magnetic ions. The spin $S$ of the magnetic ion was also replaced by an (composition dependent) effective value $S_{0}\left(S_{0}<S\right)$ since a significant fraction of the magnetic ions does not contribute to the net magnetization.

The parameters $T_{0}$ and $S_{0}$ have been obtained from the experimentally measured magnetic susceptibility of the samples at various temperatures and magnetic fields. The values of $x_{\text {eff }}=x S_{0} / S$ are 0.009 for $x=0.01$ and 0.03 for $x=0.05$, respectively. The parameter $T_{0}$ is $0.9 \mathrm{~K}$ and $1.9 \mathrm{~K}$ for $x=0.01$ and 0.05 , respectively. We used a value of $220 \mathrm{meV}$ [10] for the $s-d$ exchange constant. The donor Bohr radius value is $50 \AA$ for $x=0.01$ and $45 \AA$ for $x=0.05$, respectively [10]. The values of $\Delta N_{\mathrm{D}}$ are taken from the Hall-effect measurements. There are no fitting parameters in our calculations. We find that the BMP theory [5] describes correctly the saturation of the BMP magnetization with increasing magnetic field. This effect had not been observed in earlier experiments. The correlation between the BMP magnetization and the light-induced persistent donor concentration is consistent with the negative- $U$ defect model for $D X$-like centers in II-VI semiconductors [11].

We thank Prof. J. Gaj for useful comments. This work was partially supported by Project MAT 94-0043 of Comisión Interministerial de Ciencia y Tecnología (CICYT).

\section{References}

[1] M. Nawrocki, R. Planel, G. Fishman, R. Gałazka, Phys. Rev. Lett. 46, 735 (1981); D. Heiman, Y. Shapira, S. Foner, Solid State Commun. 45, 899 (1983).

[2] A. Golnik, J. Ginter, J.A. Gaj, J. Phys. C 16, 6073 (1983); C.A. Huber, J.A. Perez, T.E. Huber, Phys. Rev. B 36, 5933 (1987).

[3] T. Dietl, J. Antoszewski, L. Świerkowski, Physica B 117\&118, 491 (1983).

[4] T. Dietl, J. Spałek, Phys. Rev. Lett. 48, 355 (1982); Phys. Rev. B 28, 1548 (1983).

[5] P.A. Wolf, in: Diluted Magnetic Semiconductors, in series Semiconductors and Semimetals, Vol. 25, Eds. R.K. Willardson, A.C. Beers, Academic Press, London 1987, p. 413.

[6] W.E. Hagston, T. Stirner, J. P. Goodwin, P. Harrison, Phys. Rev. B 50, 5255 (1994); W.E. Hagston, T. Stirner, P. Harrison, O.F. Holbrook, J.P. Goodwin, ibid. 50, 5264 (1994).

[7] T. Wojtowicz, S. Koleśnik, I. Miotkowski, J.K. Furdyna, Acta Phys. Pol. A 82, 637 (1992); T. Wojtowicz, S. Koleśnik, I. Miotkowski, J.K. Furdyna, Phys. Rev. Lett. 70, 2317 (1993).

[8] I. Terry, T. Penney, S. von Molnár, J.M. Rigotty, P. Becla, Solid State Commun. 84, 235 (1992); N.G. Semaltianos, G. Karczewski, T. Wojtowicz, J.K. Furdyna, Phys. Rev. B 47, 12540 (1993). 
[9] J.A. Gaj, R. Planel, G. Fishman, Solid State Commun. 29, 435 (1979).

[10] D.L. Peterson, D.U. Bartholomew, U. Dębska, A.K. Ramdas, S. Rodriguez, Phys. Rev. B 32, 323 (1985).

[11] D.J. Chadi, Phys. Rev. Lett. 72, 534 (1994). 